\title{
Raman Response in the Nematic Phase of FeSe
}

\author{
Mattia Udina, ${ }^{1}$ Marco Grilliø, ${ }^{1}$ Lara Benfatto $\odot,{ }^{1, *}$ and Andrey V. Chubukov $\odot^{2}$ \\ ${ }^{1}$ Department of Physics and ISC-CNR, "Sapienza" University of Rome, P.le A. Moro 5, 00185 Rome, Italy \\ ${ }^{2}$ School of Physics and Astronomy, University of Minnesota, Minneapolis, Minnesota 55455, USA
}

(Received 29 August 2019; revised manuscript received 21 January 2020; accepted 21 April 2020; published 12 May 2020)

\begin{abstract}
Raman experiments on bulk FeSe revealed that the low-frequency part of the $B_{1 g}$ Raman response $R_{B 1 g}(\Omega)$, which probes nematic fluctuations, rapidly decreases below the nematic transition at $T_{n} \sim 85 \mathrm{~K}$. Such behavior is expected when a gap opens up and at a first glance is inconsistent with the fact that FeSe remains a metal below $T_{n}$. We argue that the drop of $R_{B 1 g}(\Omega)$ can be ascribed to the fact that the nematic order drastically changes the orbital content of low-energy excitations near hole and electron pockets, making them nearly mono-orbital. In this situation, the $B_{1 g}$ Raman response gets reduced by the same vertex corrections that enforce charge conservation in the symmetric Raman channel. The reduction holds at low frequencies and gives rise to gaplike behavior of $R_{B 1 g}(\Omega)$. We also show that the enhancement of the $B_{1 g}$ Raman response near $T_{n}$ is consistent with the sign change of the nematic order parameter between hole and electron pockets.
\end{abstract}

DOI: 10.1103/PhysRevLett.124.197602

Introduction.-Bulk Fe-chalcogenide FeSe has recently attracted a lot of attention due to a peculiar property, not seen in other Fe-based superconductors - the emergence of a nematic order (a spontaneous breaking of $C_{4}$ lattice symmetry down to $C_{2}$ ) below $T_{n} \sim 85 \mathrm{~K}$, without antiferromagnetism nearby [1-4]. Extensive STM and ARPES studies [5-18] revealed a sizeable deformation of hole and electron Fermi surfaces (FSs) below $T_{n}$, which are well reproduced by introducing an electronic orbital splitting [8,11,19-21].

A remarkable indication of the electronic nature of the nematic transition in FeSe has been provided by Raman measurements [22-25]. In a metal, the Raman response probes densitylike fluctuations at a finite frequency $\Omega$ and vanishing momenta $q$, modulated by a form factor, which depends on relative polarizations of the incoming and outgoing light and transforms according to the point-group representation of the crystal [26]. When the form-factor is constant as, e.g., in the fully symmetric channel in a singleband system, the Raman response is proportional to the density correlator at $q=0$ and finite $\Omega$, and it vanishes because fermionic density is a conserved quantity [4,26-34]. The Raman response which probes electronic nematic correlations in FeSe has the nonsymmetric $B_{1 g}$ symmetry [35], so it is finite in the metal because no conservation law applies [4,29-32].

Above the nematic transition, the measured profile of the Raman intensity $R_{B 1 g}(\Omega)$ at small frequencies is well approximated $[4,29,36]$ by a relaxational form $R_{B_{1 g}}(\Omega) \propto$ $\Omega /\left(\Omega^{2}+4 \gamma_{\text {eff }}^{2}\right)$, where the effective scattering rate $\gamma_{\text {eff }}$ is either due to impurities [4] or to electron-electron interaction [31,32,37]. Critical fluctuations give [29,36,37] $\gamma_{\text {eff }} \propto T-T_{n}$, so that as $T$ approaches $T_{n}$ the peak is expected to move to a lower frequency and to gain intensity. Both results agree with the data $[4,22-25]$. However, below the nematic transition, the data show that $R_{B 1 g}(\Omega)$ rapidly drops at $\Omega \lesssim 200 \mathrm{~cm}^{-1}$. Such behavior is expected when quasiparticles acquire a finite gap (e.g., in a superconductor $[33,34]$ ), but $\mathrm{FeSe}$ remains a metal at $T<T_{n}$, with deformed, but still sizable hole and electron pockets. We argue that the Fermi surface deformation has little effect on the Raman response, and the origin of the gaplike behavior is the change of the orbital composition of the pockets below $T_{n}$.

The outline of our reasoning is as follows. In the tetragonal phase, FeSe has two nearly circular hole pockets at the zone center $\Gamma$ and two electron pockets at $M=(\pi, \pi)$ (in the $2 \mathrm{Fe}$ Brillouin-zone notation), split by spin-orbit coupling into inner and outer pockets. The hole and inner electron pockets are made of $d_{x z}$ and $d_{y z}$ orbitals, and the outer electron pocket has predominantly $d_{x y}$ character [38]. The $B_{1 g}$ Raman vertex distinguishes between $d_{x z}$ and $d_{y z}$ orbitals, and it is well approximated by $\Gamma_{B_{1 g}}(k)=$ $n_{x z}(k)-n_{y z}(k)$, where $n=d^{\dagger} d$, and $d^{\dagger}, d$ are creation and annihilation operators for the corresponding orbitals $[4,23,39]$. In the band basis, $\Gamma_{B_{1 g}}(k)$ in the tetragonal phase has pure $d$-wave symmetry, e.g., near the outer hole pocket $\Gamma_{B_{1 g}}=d_{h}^{\dagger} d_{h} \cos 2 \theta$, where $d_{h}^{\dagger}, d_{h}$ are band operators and $\theta$ is the angle along the pocket. The nematicity breaks $C_{4}$ symmetry between the orbital occupations and induces an additional term $\Delta(k)\left[n_{x z}(k)-n_{y z}(k)\right]$ in the Hamiltonian [40]. This elongates hole and inner electron pockets in the directions set by the signs of $\Delta(k)$ at $\Gamma$ and at $M$ points, $\Delta_{h}$ and $\Delta_{e}$ (Refs. [8,11,19-21,41]) and changes the orbital 
content of the pockets. This affects $\Gamma_{B_{1 g}}(k)$, which develops an angle-independent component, proportional to $\Delta_{h(e)}$. In FeSe, the pockets are small, and the orbital content changes quite drastically. Calculations [8,19-21] and polarized ARPES data $[14,16]$ show that deep inside the nematic phase the outer hole pocket becomes predominantly $d_{x z}$ and the inner electron pocket becomes predominantly $d_{y z}$. In this situation, the angle-independent component of $\Gamma_{B_{1 q}}$ becomes the dominant one. Then $R_{B 1 g}(\Omega)$ drops because $B_{1 g}$ Raman susceptibility becomes nearly identical to the susceptibility in the symmetric density channel, and the latter vanishes at $q=0$ and finite $\Omega$ by charge conservation [4,26-34]. The reduction holds at $\Omega \leq 2-3 \Delta_{h(e)}$. At larger $\Omega$ electronic excitations recover the same orbital character of the tetragonal phase and $R_{B 1 g}(\Omega)$ rapidly increases.

The nematic order can originate from a $d$-wave Pomeranchuk instability [30,41,42] or from composite spin fluctuations $[8,43,44]$. The microscopic origin of the nematic order parameter is not important for our purposes-we will just compare the Raman response in the presence and absence of $\Delta_{h(e)}$. At the computational level, the reduction of $R_{B 1 g}(\Omega)$ is associated with the effect of impurity-induced vertex corrections, which must be included along with the fermionic self-energy (Ref. [45]).

Orbital composition of the pockets.-The low-energy states near the hole pockets are described, in $1 \mathrm{Fe}$ zone, by the effective Hamiltonian [46]

$$
H=\sum_{\mathbf{k}, \sigma, \sigma^{\prime}} \Psi_{\mathbf{k}, \sigma}^{\dagger} \hat{K} \Psi_{\mathbf{k}, \sigma^{\prime}}
$$

where $\Psi \equiv\left(d_{y z}, d_{x z}\right)$ is a spinor in the orbital space, and $\hat{K}=\left[h_{0} \tau_{0}+h_{1} \tau_{1}+\left(h_{3}-\Delta_{h}\right) \tau_{3}\right] \sigma_{\sigma \sigma^{\prime}}^{(0)}+\eta \tau_{2} \sigma_{\sigma \sigma^{\prime}}^{(3)}$. The hopping integrals in momentum space are $h_{0}(\mathbf{k})=\epsilon_{0}-k^{2} /(2 m)$, $h_{1}(\mathbf{k})=-2 c k_{x} k_{y}$, and $h_{3}(\mathbf{k})=b\left(k_{x}^{2}-k_{y}^{2}\right)$. ( $\epsilon_{0}$ includes the static self-energy that accounts for the shrinking of the hole pockets $[8,21,44]), \eta$ is the spin-orbit $(\mathbf{L} \cdot \mathbf{S})$ coupling, and $\Delta_{h}=\Delta_{h}(T)$ is the magnitude of the nematic order, which we set to be positive in accordance with the experimental findings [5-18]. To simplify the presentation, in analytical formulas below we set $b=c>0$, in which case the pocket in the tetragonal phase is circular. For numerical calculations we choose the hopping parameters which match the data on FeSe. The transformation from the orbital to the band basis is described by the unitary matrix with components $u_{\mathbf{k}}^{2}=\cos ^{2} \bar{\theta}_{\mathbf{k}}, v_{\mathbf{k}}^{2}=\sin ^{2} \bar{\theta}_{\mathbf{k}}$, where

$$
\cos 2 \bar{\theta}_{\mathbf{k}}=\frac{b k^{2} \cos 2 \theta-\Delta_{h}}{\sqrt{b^{2} k^{4}+\Delta_{h}^{2}-2 b k^{2} \Delta_{h} \cos 2 \theta+\eta^{2}}},
$$

and $\theta$ is the angle along the hole pocket. At $\eta=\Delta_{h}=0$, two hole bands necessary cross the Fermi level and form the inner and the outer hole pockets. When $\eta$ or $\Delta_{h}$ (or both) are nonzero, the inner pocket gets smaller and may sink below the Fermi level. In FeSe, only the outer hole pocket has been detected in the nematic state, and in analytical treatment we focus on this band. The Hamiltonian in the band basis is $H=\sum_{\mathbf{k}} E_{\mathbf{k}} d_{h, \mathbf{k}}^{\dagger} d_{h, \mathbf{k}}$ with $E_{\mathbf{k}}=$ $\epsilon_{0}-k^{2} /(2 m)+\sqrt{b^{2} k^{4}+\Delta_{h}^{2}-2 b k^{2} \Delta_{h} \cos 2 \theta+\eta^{2}}$. The weight of $d_{x z}$ and $d_{y z}$ orbitals is given by $v_{\mathbf{k}}^{2}=$ $\left(1-\cos 2 \bar{\theta}_{\mathbf{k}}\right) / 2$ and $u_{\mathbf{k}}^{2}=\left(1+\cos 2 \bar{\theta}_{\mathbf{k}}\right) / 2$, respectively. In the tetragonal phase $\cos 2 \bar{\theta}_{\mathbf{k}} \propto \cos 2 \theta$, and $d_{x z}$ and $d_{y z}$ orbitals equally contribute to band excitations. In the nematic phase, the weight depends on the ratio $\lambda_{h}=$ $\Delta_{h} /\left(b k^{2}\right)$. When $\lambda_{h}$ is small, the two orbitals still contribute nearly equally. However, when $\lambda_{h} \geq 1, \cos 2 \bar{\theta}_{\mathbf{k}} \approx-1$, independent on $\theta$. In this limit, the hole pocket is made almost entirely of $d_{x z}$ orbitals. Recent polarized ARPES study of FeSe did find $[14,16]$ that the weight of the $d_{x z}$ is over $80 \%$ along the hole pocket. This holds when $\lambda_{h} \sim$ 0.7 (Ref. [20]).

A very similar result holds for the inner electron pocket near $(\pi, \pi)$. In the tetragonal phase, this pocket is almost circular, and composed equally of $d_{x z}$ and $d_{y z}$ orbitals. The evolution of the orbital composition of this pocket below $T_{n}$ is quite similar to that of the hole pocket (see [47]), except that $\Delta_{e}<0$ [5-18]. We can still model it with Eq. (1), provided that $m$ and $b$ become negative to describe an electronlike dispersion. By replacing $b \rightarrow|b|$ and $\Delta_{h}$ with $\left|\Delta_{e}\right|$ in Eq. (2), in the tetragonal phase it is still $\cos 2 \bar{\theta}_{\mathbf{k}}=\cos 2 \theta$. However, since now $u_{\mathbf{k}}^{2}=\sin ^{2} \bar{\theta}_{\mathbf{k}}$ and $v_{\mathbf{k}}^{2}=\cos ^{2} \bar{\theta}_{\mathbf{k}}$ the angular dependence of the orbital composition at the electron pocket is reversed. In addition, in some distinction to the hole pocket, for a large $\left|\Delta_{e}\right|$, the orbital content becomes predominantly $d_{y z}$ for most of $\theta$, but for $\theta=0$ it remains $d_{x z}[14,16]$. The outer electron pocket has instead almost entirely $x y$ character both above and below $T_{n}$ [38]. We follow earlier works $[11,19,48]$, which argued that fermions in the $x y$ band have a small residue, and neglect processes involving $d_{x y}$ fermions.

The Raman response in the nematic phase.-The change of the orbital composition of the excitations has a profound effect on the Raman response. The $B_{1 g}$ Raman density in the band basis is expressed as [47] $\rho_{h(e)}^{B_{1 g}}(\mathbf{q})=$ $\sum_{\mathbf{k}_{i}}(-1)^{i} d_{h(e), \mathbf{k}_{i}}^{\dagger} d_{h(e), \mathbf{k}_{i}+\mathbf{q}} \cos 2 \bar{\theta}_{\mathbf{k}_{i}}$, where $\mathbf{k}_{1}$ is near $\Gamma$ and $\mathbf{k}_{2}$ is near $M$. The factor $(-1)^{i}$ is the consequence of the fact that the orbital content flips between the outer hole pocket and the inner electron pocket. The density-density interaction, which favors a sign-changing nematic order, is interpocket repulsion $H_{I}=U_{e h} \sum_{\mathbf{q}} \rho_{h}^{B_{1 g}}(\mathbf{q}) \rho_{e}^{B_{1 g}}(\mathbf{q})[24,49]$. In analogy with $s^{ \pm}$superconductivity, a positive $U_{e h}>0$ favors a sign change between $\Delta_{e} \equiv\left\langle\rho_{e}^{B_{1 g}}(\mathbf{q}=0)\right\rangle$ and $\Delta_{h} \equiv\left\langle\rho_{h}^{B_{1 g}}(\mathbf{q}=0)\right\rangle$. We assume that interpocket repulsion is larger than intrapocket nematic interactions. Summing up the ladder series of corrections to the $B_{1 g}$ Raman vertex, we 


\begin{tabular}{|c|c|}
\hline$R_{B_{1 g}} \propto \operatorname{Im}\left[\frac{\chi_{h}+\chi_{e}+2 U_{e h} \chi_{h} \chi_{e}}{1-U_{e h}^{2} \chi_{h} \chi_{e}}\right]$ \\
\hline Tetragonal & Nematic \\
\hline$\chi_{h(e)}=\chi_{h(e)}^{s e}$ & $\chi_{h(e)}=\chi_{h(e)}^{s e}+\chi_{h(e)}^{v}$ \\
\hline & \\
$\chi_{h(e)}^{s e}=$ & \\
\hline
\end{tabular}

FIG. 1. Diagrammatic representation of the Raman susceptibility for angle-independent impurity scattering. Here single (double) lines denote bare (dressed) electronic Green's functions, the dashed line with a cross denotes the impurity potential. In the tetragonal phase, the Raman vertex (denoted by a dot) has pure $d$ wave form, and vertex corrections due to impurity scattering vanish. In the nematic phase, the vertex has both $d$ - and $s$-wave components, and the $s$-wave part is eliminated by vertex corrections.

find the total contribution from hole and electron pockets in the form [47]:

$R_{B_{1 g}}(\Omega) \propto \operatorname{Im}\left[\frac{\chi_{h}(\Omega)+\chi_{e}(\Omega)+2 U_{e h} \chi_{h}(\Omega) \chi_{e}(\Omega)}{1-U_{e h}^{2} \chi_{h}(\Omega) \chi_{e}(\Omega)}\right]$,

where $\chi_{h(e)}(\Omega)=\chi_{h(e)}(q=0, \Omega)$ is the fully dressed particle-hole susceptibility with Raman side vertices $\cos 2 \bar{\theta}_{\mathbf{k}}$, where $\mathbf{k}$ is near $\Gamma$ and $M$, respectively. For $\chi_{h}(\Omega)=$ $\chi_{e}(\Omega)=\chi(\Omega), R_{B_{1 g}}(\Omega) \propto \operatorname{Im}\left[\chi(\Omega) / 1-U_{e h} \chi(\Omega)\right]$. Since $\chi(\Omega)>0$, the observed enhancement of $R_{B_{1 g}}(\Omega)$ near $T_{n}$ occurs only if interpocket $U_{e h}>0$. This is consistent with the sign change between $\Delta_{h}$ and $\Delta_{e}$ in the nematic phase.

The diagrams for Raman susceptibilities $\chi_{h(e)}(\Omega)$ are presented in Fig. 1. The dressed bubble is $\chi_{h(e)}(\Omega)=$ $\chi_{h(e)}^{s e}(\Omega)+\chi_{h(e)}^{v}(\Omega)$, where $\chi_{h(e)}^{s e}(\Omega) \propto 2 i \gamma_{h(e)} /\left(\Omega+2 i \gamma_{h(e)}\right)$ is the particle-hole bubble made of fermions with damping rate $\gamma_{h(e)}$ (assumed to be angle independent), and $\chi_{h(e)}^{v}(\Omega)$ accounts for vertex corrections. In the tetragonal phase, $\cos 2 \bar{\theta}_{\mathbf{k}}=\cos 2 \theta$, and vertex corrections due to impurity scattering vanish because $\oint d \theta \cos 2 \bar{\theta}_{\mathbf{k}}=0$ and $\chi_{h(e)}(\Omega)=$ $\chi_{h(e)}^{s e}(\Omega)$. In the nematic phase, $R_{B 1 g}(\Omega)$ changes because the pocket becomes elliptical (or even peanutlike), and because the orbital content of particle-hole excitations changes. We verified [see Fig. 2(a)] that the change of the shapes of the pockets actually somewhat enhances Raman susceptibility. However, the change of orbital content strongly reduces $R_{B_{1 g}}$. Indeed, in the nematic phase
Without vertex corrections
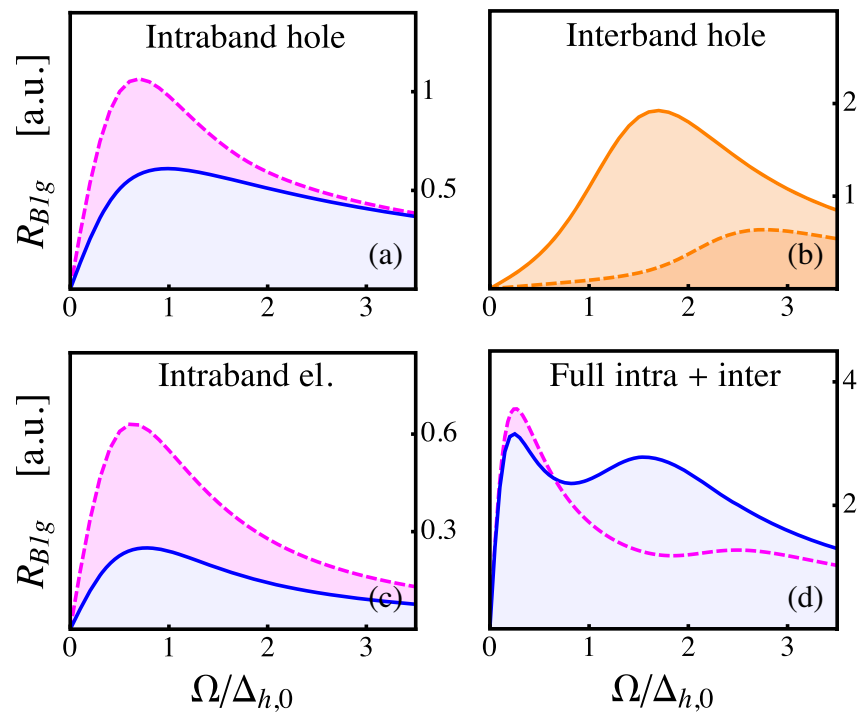

FIG. 2. Raman intensity in $B_{1 g}$ channel, obtained neglecting vertex corrections. Solid (dashed) lines denote the results in the tetragonal (nematic) state. The frequency is in units of $\Delta_{h, 0}=\Delta_{h}(T=0)$. (a),(b) Intraband (a) and interband (b) response from the hole pockets. (c) Intraband response for the inner electron pocket. (d) Full intraband response, obtained using Eq. (3), along with the interband part, panel (b). Interband response here is without RPA resummation, since the effect of the latter is rather mild, see [47]. We set $\Delta_{h .0}=15 \mathrm{meV}$, $\Delta_{e}(T)=-1.3 \Delta_{h}(T)$ and $\gamma_{h}=\gamma_{e}=6 \mathrm{meV}$. The band parameters are listed in the Supplemental Material [47]. The double peak structure of $R_{B 1 g}(\Omega)$ in the tetragonal phase is consistent with Refs. [4,22].

$\cos 2 \bar{\theta}_{\mathbf{k}}$ acquires an angle-independent, $s$-wave component [see Eq. (2)], i.e., $\cos 2 \bar{\theta}_{\mathbf{k}} \approx \Gamma_{s ; \mathbf{k}}+\Gamma_{d ; \mathbf{k}} \cos 2 \theta$. For the $d$-wave component, vertex corrections are still irrelevant, but for $s$-wave component they are nonzero and have to be included. Summing up ladder series of vertex corrections (see Fig. 1) we obtain [47]

$\chi_{h(e)}^{s e}(\Omega)=N_{F, h(e)} \frac{2 i \gamma_{h(e)}}{\Omega+2 i \gamma_{h(e)}}\left(\Gamma_{s, h(e)}^{2}+\Gamma_{d, h(e)}^{2}\right)$,

$\chi_{h(e)}^{v}(\Omega)=-N_{F, h(e)} \frac{2 i \gamma_{h(e)}}{\Omega+2 i \gamma_{h(e)}} \Gamma_{s, h(e)}^{2}$,

where $N_{F, h(e)}$ is the density of states at the Fermi level. Adding up the two terms we find that the $s$-wave contribution expectedly cancels out. As a result, Raman intensity remains proportional to $\Gamma_{d, h(e)}^{2}$. For identical pockets, $N_{F, h(e)}=N_{F}, \gamma_{h(e)}=\gamma, \Gamma_{d, h(e)}=\Gamma_{d}$ we obtain

$$
R_{B 1 g}(\Omega) \propto \Gamma_{d}^{2} \frac{\Omega \gamma}{\Omega^{2}+4 \gamma^{2}\left(1-U_{e h} N_{F} \Gamma_{d}^{2}\right)^{2}} .
$$


For $\Gamma_{d, h} \neq \Gamma_{d, e}$ the structure of $R_{B 1 g}(\Omega)$ is more complicated [47], but it still only involves the $d$-wave components of Raman vertices. When the hole pocket becomes almost entirely $d_{x z}$ and the inner electron pocket becomes $d_{y z}, \Gamma_{s, h(e)} \approx \mp 1$, and $\Gamma_{d, h(e)}$ are small. Then $R_{B 1 g}(\Omega)$ is strongly reduced.

The reduction holds only at small frequencies. At larger $\Omega$, typical momenta $\mathbf{k}$ in the polarization bubbles move away from the corresponding $k_{F, h(e)}, \lambda_{h(e)} \propto \Delta_{h(e)} /\left(b_{h(e)} k^{2}\right)$ get smaller, and $\Gamma_{d, h(e)}$ increase. This can be well captured by including explicitly the dependence of $\Gamma_{s, h(e)}$ and $\Gamma_{d, h(e)}$ on frequency:

$$
\begin{aligned}
\Gamma_{s, h(e)}(\Omega) & =\mp \int \frac{d \theta k d k \delta}{(2 \pi)^{2}} \frac{\delta\left(\Omega-E_{\mathbf{k}, h(e)}\right)}{N_{F, h(e)}(\Omega)} \cos 2 \bar{\theta}_{\mathbf{k}}, \\
\Gamma_{s, h(e)}^{2}(\Omega)+\Gamma_{d, h(e)}^{2}(\Omega) & =\int \frac{d \theta k d k \delta\left(\Omega-E_{\mathbf{k}, h(e)}\right)}{(2 \pi)^{2}} \frac{\left.\cos 2 \bar{\theta}_{\mathbf{k}}\right)^{2},}{N_{F, h(e)}(\Omega)}
\end{aligned}
$$

where $N_{F, h(e)}(\Omega)=\int\left[(d \theta k d k) /(2 \pi)^{2}\right] \delta\left(\Omega-E_{\mathbf{k}, h(e)}\right)$ is the frequency-dependent density of states, and the integration is near either hole or electron pocket. The Raman response $R_{B 1 g}(\Omega)$ is given by Eq. (5) with $\Gamma_{d, h(e)}=\Gamma_{d, h(e)}(\Omega)$. As $\Omega$ increases, the relevant $k$ in Eq. (6) increases, $\lambda_{h(e)} \ll 1$, and for a generic $\theta$ one recovers $\cos 2 \bar{\theta}_{\mathbf{k}}=\cos 2 \theta$. In this situation the $s$-wave component of $\cos 2 \bar{\theta}_{\mathbf{k}}$ drops out, and the Raman response becomes the same as in the tetragonal phase. Note in passing that long-range Coulomb interaction does not affect $R_{B 1 g}(\Omega)$ because the "mixed" bubble, with $B_{1 g}$ Raman vertex on one side and a constant ( $s$-wave) vertex on the other, vanishes due to vertex corrections, even when both $\Gamma_{s, h(e)}$ and $\Gamma_{d, h(e)}$ are nonzero (Refs. [33,34]). Also, the $A_{1 g}$ Raman response vanishes in both tetragonal and nematic phases despite the mixing between $s$-wave and $d$-wave components, since the $A_{1 g}$ Raman vertex is always a constant. We show this explicitly in the Supplemental Material [47].

Numerical calculations.-To set up a more realistic comparison with the experimental data we computed $R_{B 1 g}(\Omega)$ in the tetragonal and nematic phases numerically 47]], using parameters appropriate to reproduce the band dispersion of $\mathrm{FeSe}$, as measured by ARPES. We first show, in Fig. 2, the result for Raman intensity without vertex corrections, i.e., with $\chi_{h(e)}=\chi_{h(e)}^{s e}$ [Eq. (5) with $\left.\Gamma_{d, h(e)} \approx 1\right]$. In this approximation, the difference between $R_{B 1 g}(\Omega)$ for $T>T_{n}$ and $T<T_{n}$ is only due to changes in the shapes of the pockets. While the interband part moves to higher frequencies due to the nematic splitting between the bands, the intraband contributions actually get slightly enhanced below $T_{n}$. In [47] we show how $R_{B 1 g}(\Omega)$ changes if we additionally vary $U_{e h}$ to model the reduction of $U_{e h} N_{F}$ below $T_{n}$. $R_{B 1 g}(\Omega)$ decreases, but does not show gaplike behavior. The situation changes drastically when we include vertex corrections. We show the result for the full intraband $R_{B 1 g}(\Omega)$ in Fig. 3(c) and for the total $R_{B 1 g}(\Omega)$ with both intraband and interband contributions in [47]. We used a mean-field-like temperature dependence of $\Delta_{h(e)}(T)$, which is in good agreement with the observed temperature evolution of the band dispersion below $T_{n}$ [8]. We see the behavior that we just outlined. Namely, at small $\Omega$ the Raman peak progressively loses intensity as $T$ is lowered below $T_{n}$. The peak survives over some range of $T<T_{n}$, but eventually $R_{B 1 g}(\Omega)$ develops a gaplike behavior at small frequencies. The fine details of the evolution of $R_{B 1 g}(\Omega)$ below $T_{n}$ somewhat depend on the band modeling (see the Supplemental Material [47]), but the overall suppression of the Raman intensity is quite robust and agrees with the data $[22,24,25]$.

Summary and discussion. - In this work we have shown that the observed gaplike behavior of the $B_{1 g}$ Raman response in FeSe, i.e., in a nematic metal, is a direct consequence of the change of orbital composition of the pockets, which become nearly mono-orbital at $T \ll T_{n}$. The Raman intensity $R_{B 1 g}(\Omega)$ contains only the nonsymmetric $d$-wave part of the form factor. However, the change in the orbital content of the pockets induces a strong mixing between $d$-wave and $s$-wave channels. When pockets become nearly mono-orbital, the angle-independent

\section{With vertex corrections}

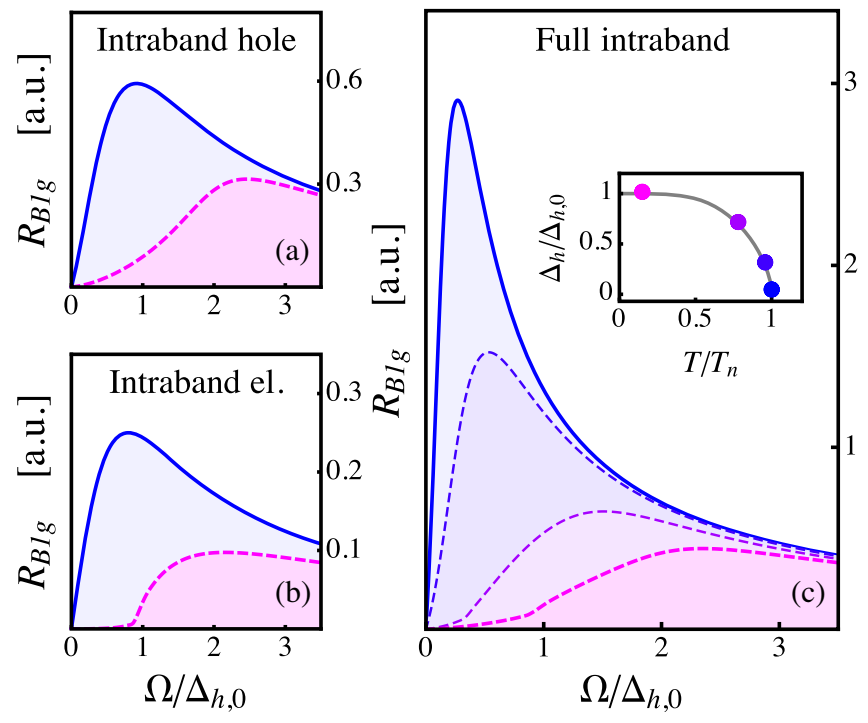

FIG. 3. Intraband Raman response Eq. (3) including vertex corrections. Solid (dashed) lines denote the response in the tetragonal (nematic) state. (a),(b) Intraband response from the outer hole pocket and the inner electron pocket at the lowest $T$. (c) The full intraband response at $T \leq T_{n}$ shown in the inset. The response at $T \ll T_{n}$ nearly vanishes at small $\Omega$ and recovers its form in the tetragonal phase at $\Omega \geq 3 \Delta_{h, 0}$. The system parameters are the same as in Fig. 2. The total $R_{B 1 g}(\Omega)$, including interband contribution, is shown in [47]. 
component of the $B_{1 g}$ Raman form factor rises at the expense of the original $\cos 2 \theta$ component, and the $B_{1 g}$ Raman intensity drops. We also found that the increase of $R_{B_{1 g}}$ near $T_{n}$ holds only if the nematic order changes sign between hole and electron pockets.

To put our results in a broader context, we note that so far the orbital reconstruction of fermionic excitations in FeSe due to nematicity has been analyzed in polarized ARPES measurements $[14,16]$, which are surface sensitive. Our work demonstrates that the Raman spectroscopy, which is a bulk probe, is also capable to detect orbital transmutation in iron-based systems. It then will be particularly useful in systems where detailed ARPES data are not available. We note that our findings are inconsistent with the conjecture of orbital-dependent spectral weights, put forward in Refs. [11,19,50-52]. Within that conjecture, the orbital weights are assumed to get rescaled by interactions in such a way that, e.g., the outer hole pocket does not become nearly entirely $d_{x z}$ in the nematic phase. In this situation, the Raman response would not show strong suppression at low frequency in the nematic phase, which is in disagreement with the data.

We are thankful to B. Andersen, A. Bohmer, G. Blumberg, M. Christensen, R. Fernandes, P. Hirschfeld, Y. Gallais, J. Kang, A. Klein, A. Kreisel, I. Paul, A. Sacuto, and $\mathrm{H}$. Yamase for useful discussions. This work has been supported by the Office of Basic Energy Sciences, U.S. Department of Energy, under Award No. DE-SC0014402, by the Italian MAECI under the Italian-India collaborative Project No. SUPERTOP-PGR04879, by the Italian MIUR project PRIN 2017 No. 2017Z8TS5B, and by Regione Lazio (L. R. 13/08) under project SIMAP. A. V. C. thanks for hospitality the Sapienza University of Rome, where this work was initiated.

*lara.benfatto@roma1.infn.it

[1] A. E. Böhmer and A. Kreisel, J. Phys. Condens. Matter 30, 023001 (2018).

[2] A. I. Coldea and M. D. Watson, Annu. Rev. Condens. Matter Phys. 9, 125 (2018).

[3] R. Fernandes and A. V. Chubukov, Rep. Prog. Phys. 80, 014503 (2017).

[4] Y. Gallais and I. Paul, C.R. Phys. 17, 113 (2016).

[5] Y. Suzuki, T. Shimojima, T. Sonobe, A. Nakamura, M. Sakano, H. Tsuji, J. Omachi, K. Yoshioka, M. KuwataGonokami, T. Watashige, R. Kobayashi, S. Kasahara, T. Shibauchi, Y. Matsuda, Y. Yamakawa, H. Kontani, and K. Ishizaka, Phys. Rev. B 92, 205117 (2015).

[6] M. D. Watson, T. K. Kim, A. A. Haghighirad, N. R. Davies, A. McCollam, A. Narayanan, S. F. Blake, Y. L. Chen, S. Ghannadzadeh, A. J. Schofield, M. Hoesch, C. Meingast, T. Wolf, and A. I. Coldea, Phys. Rev. B 91, 155106 (2015).

[7] M. D. Watson, T. K. Kim, L. C. Rhodes, M. Eschrig, M. Hoesch, A. A. Haghighirad, and A. I. Coldea, Phys. Rev. B 94, 201107(R) (2016).
[8] L. Fanfarillo, J. Mansart, P. Toulemonde, H. Cercellier, P. Le Fèvre, F. Bertran, B. Valenzuela, L. Benfatto, and V. Brouet, Phys. Rev. B 94, 155138 (2016).

[9] M. D. Watson, A. A. Haghighirad, L. C. Rhodes, M. Hoesch, and T. K. Kim, New J. Phys. 19, 103021 (2017).

[10] L. C. Rhodes, M. D. Watson, A. A. Haghighirad, M. Eschrig, and T. K. Kim, Phys. Rev. B 95, 195111 (2017).

[11] P. O. Sprau, A. Kostin, A. Kreisel, A. E. Böhmer, V. Taufour, P. C. Canfield, S. Mukherjee, P. J. Hirschfeld, B. M. Andersen, and J. C. S. Davis, Science 357, 75 (2017).

[12] A. Fedorov, A. Yaresko, T. K. Kim, Y. Kushnirenko, E. Haubold, T. Wolf, M. Hoesch, A. Gruneis, B. Buechner, and S. V. Borisenko, Sci. Rep. 6, 36834 (2016).

[13] Y. S. Kushnirenko, A. A. Kordyuk, A. V. Fedorov, E. Haubold, T. Wolf, B. Büchner, and S. V. Borisenko, Phys. Rev. B 96, 100504(R) (2017).

[14] L. C. Rhodes, M. D. Watson, A. A. Haghighirad, D. V. Evtushinsky, M. Eschrig, and T. K. Kim, Phys. Rev. B 98, 180503(R) (2018).

[15] T. Hashimoto, Y. Ota, H. Q. Yamamoto, Y. Suzuki, T. Shimojima, S. Watanabe, C. Chen, S. Kasahara, Y. Matsuda, T. Shibauchi, K. Okazaki, and S. Shin, Nat. Commun. 9, 282 (2018).

[16] D. Liu et al., Phys. Rev. X 8, 031033 (2018).

[17] M. Yi et al., Phys. Rev. X 9, 041049 (2019).

[18] S. Huh et al., arXiv:1903.08360.

[19] A. Kreisel, B. M. Andersen, P. O. Sprau, A. Kostin, J. C. Seamus Davis, and P. J. Hirschfeld, Phys. Rev. B 95, 174504 (2017).

[20] J. Kang, R. M. Fernandes, and A. Chubukov, Phys. Rev. Lett. 120, 267001 (2018).

[21] L. Benfatto, B. Valenzuela, and L. Fanfarillo, npj Quantum Mater. 3, 56 (2018).

[22] P. Massat, D. Farina, I. Paul, S. Karlsson, P. Strobel, P. Toulemonde, M.-A. Méasson, M. Cazayous, A. Sacuto, S. Kasahara, T. Shibauchi, Y. Matsuda, and Y. Gallais, Proc. Natl. Acad. Sci. U.S.A. 113, 9177 (2016).

[23] V. K. Thorsmolle, M. Khodas, Z. P. Yin, C. Zhang, S. V. Carr, P. Dai, and G. Blumberg, Phys. Rev. B 93, 054515 (2016).

[24] W. L. Zhang, S. F. Wu, S. Kasahara, T. Shibauchi, Y. Matsuda, and G. Blumberg, arXiv:1710.09892.

[25] A. Baum, H. N. Ruiz, N. Lazarevic, Y. Wang, T. Bohm, R. Hosseinian Ahangharnejhad, P. Adelmann, T. Wolf, Z. V. Popovic, B. Moritz, T. P. Devereaux, and R. Hackl, Commun. Phys. 2, 14 (2019).

[26] T. P. Devereaux and R. Hackl, Rev. Mod. Phys. 79, 175 (2007).

[27] T. P. Devereaux and D. Einzel, Phys. Rev. B 51, 16336 (1995).

[28] M. V. Klein, Phys. Rev. B 82, 014507 (2010).

[29] H. Yamase and R. Zeyher, Phys. Rev. B 88, 125120 (2013).

[30] M. Tsuchiizu, Y. Ohno, S. Onari, and H. Kontani, Phys. Rev. Lett. 111, 057003 (2013).

[31] A. Klein, S. Lederer, D. Chowdhury, E. Berg, and A. Chubukov, Phys. Rev. B 97, 155115 (2018).

[32] A. Klein, S. Lederer, D. Chowdhury, E. Berg, and A. Chubukov, Phys. Rev. B 98, 041101(R) (2018).

[33] T. Cea and L. Benfatto, Phys. Rev. B 94, 064512 (2016). 
[34] S. Maiti, A. V. Chubukov, and P. J. Hirschfeld, Phys. Rev. B 96, 014503 (2017).

[35] The relevant scattering geometry is $B_{1 g}$ in 1-Fe Brillouin zone and $B_{2 g}$ in 2-Fe Brillouin zone. Throughout this Letter, we use the notation $B_{1 g}$.

[36] U. Karahasanovic, F. Kretzschmar, T. Böhm, R. Hackl, I. Paul, Y. Gallais, and J. Schmalian, Phys. Rev. B 92, 075134 (2015).

[37] F. Kretzschmar, T. Bohm, U. Karahasanovic, B. Muschler, A. Baum, D. Jost, J. Schmalian, S. Caprara, M. Grilli, C. Di Castro, J. G. Analytis, J.-H. Chu, I. R. Fisher, and R. Hackl, Nat. Phys. 12, 560 (2016).

[38] M. H. Christensen, R. M. Fernandes, and A. V. Chubukov, Phys. Rev. Research 2, 013015 (2020).

[39] A. Hinojosa, J. Cai, and A. V. Chubukov, Phys. Rev. B 93, 075106 (2016).

[40] The system may also develop $d$-wave nematic order on the outer $d_{x y}$ pocket. Such order has not been unambigiously detected in ARPES studies and we will not consider it.

[41] S. Onari, Y. Yamakawa, and H. Kontani, Phys. Rev. Lett. 116, 227001 (2016).

[42] A. V. Chubukov, M. Khodas, and R. M. Fernandes, Phys. Rev. X 6, 041045 (2016).
[43] R. M. Fernandes, A. V. Chubukov, and J. Schmalian, Nat. Phys. 10, 97 (2014)

[44] L. Fanfarillo, L. Benfatto, and B. Valenzuela, Phys. Rev. B 97, 121109(R) (2018).

[45] G. Zala, B. N. Narozhny, and I. L. Aleiner, Phys. Rev. B 64, 214204 (2001).

[46] V. Cvetkovic and O. Vafek, Phys. Rev. B 88, 134510 (2013).

[47] See the Supplemental Material at http://link.aps.org/ supplemental/10.1103/PhysRevLett.124.197602 for technical details of the calculations.

[48] L. de' Medici, Weak and Strong Correlations in Fe Superconductors (Springer Series in Materials Science, United States, 2015), Vol. 211.

[49] R.-Q. Xing, L. Classen, and A. V. Chubukov, Phys. Rev. B 98, 041108(R) (2018).

[50] H. Hu, R. Yu, E. M. Nica, J.-X. Zhu, and Q. Si, Phys. Rev. B 98, 220503(R) (2018).

[51] A. Kreisel, B. M. Andersen, and P. J. Hirschfeld, Phys. Rev. B 98, 214518 (2018).

[52] T. Chen, Y. Chen, A. Kreisel, X. Lu, A. Schneidewind, Y. Qiu, J. T. Park, T. G. Perring, J. R. Stewart, H. Cao, R. Zhang, Y. Li, Y. Rong, Y. Wei, B. M. Andersen, P. J. Hirschfeld, C. Broholm, and P. Dai, Nat. Mater. 18, 709 (2019). 